\title{
Scalar Field Therapy and Mitigation of Seizure Disorder: A Case Report
}

\author{
Noelani Apau Ludlumª, Terry Shintani ${ }^{\mathrm{a}, \mathrm{b}}$, Rosanne Harrigan ${ }^{\mathrm{a}, \mathrm{b}}$
}

\begin{abstract}
This case report describes the use of scalar field therapy in the mitigation of seizures in an autistic child. The patient is a 4-yr-10-moold girl with a 3 year history of seizure disorder and autism. She presents with a long history of a series of 6 to 8 tonic-clonic seizures on a daily basis, each lasting approximately 10 to 20 seconds long. After a series of treatments in the scalar field, her seizures were reduced to 4 to 5 partial seizures of just 1 to 2 seconds in duration. The reduction in number and duration of seizures in this case suggests that the use of scalar field therapy in the treatment of seizure disorder should be further explored. Of great importance is the potential for controlling seizures while minimizing the use of pharmaceuticals that are often debilitating and have numerous negative side effects.
\end{abstract}

Keywords: Seizures; Neurology; Scalar; Scalar field; Scalar wave; Tonic clonic; Autism; Epilepsy; Non-pharmaceutical; Therapy

\section{Introduction}

This case report describes the non-pharmaceutical reduction of seizures associated with the use of scalar field therapy in a 4-year old child with seizure disorder and autism. Seizure disorder prevalence is estimated at $350 / 100,000$ or $2-3 \%$ in children [1].

Seizure disorders in children with autism have high co-morbidity, up to $38 \%$. Autistic children have a bimodal

Manuscript accepted for publication August 21, 2012

${ }^{a}$ Department of Complementary and Alternative Medicine, John A. Burns School of Medicine, 651 Ilalo St. MEB, Honolulu, HI 96813, USA

${ }^{\mathrm{b}}$ Corresponding authors: Terry Shintani. Email: ttshintani@msn.com. Noelani Apau Ludlum. Email: napau@hawaii.edu. Rosanne Harrigan. Email: harrigan@hawaii.edu

doi: http://dx.doi.org/10.4021/jnr134w onset of epilepsy with the first peak before 5 years old and a second peak during adolescence. Seizures are much more frequent with mental retardation, and more extensive with severe mental retardation and cerebral palsy. Seizures may also contribute to autistic regression [2]. Its impact on a family can be devastating as the affected person may require round-the-clock surveillance and care for years.

Currently, the treatment approaches to seizure disorder include primarily pharmacological intervention along with dietary intervention and various surgical and physical/electronic device interventions [3]. While pharmacological intervention has had some success in managing seizure disorder, for many patients, the side effects of the pharmaceuticals such as somnolence, fatigue, depression, and cognitive effects are problematic especially for an autistic child [4]. For this reason, any non-invasive non-pharmacological approach that shows promise is an attractive adjunct to therapy and is worth investigating.

\section{Case Report}

The patient is a 4-yr-10-mo-old Polynesian, Caucasian, Asian female with a 3 year history of seizure disorder and autism. She was diagnosed by her Pediatric Neurologist. Her mental age was estimated to be at the 2 year old level. She presents with a continued series of 6 to 8 tonic-clonic seizures on a daily basis, each lasting approximately 10 to 20 seconds long as well as 8 to 12 partial or atonic seizures lasting 1 to 2 seconds long for 3 years. She was on lamictal and micelene at times caused excessive sedation. Her parents seek a way to reduce the need for medication while also reducing and controlling her seizures.

\section{History of present illness}

The patient had a normal gestation and a term vaginal birth that was uneventful. She met her developmental milestones ahead of schedule, sitting at 7 months and walking at 10 months. She appeared to develop normally until she was approximately one-year-old. In retrospect, it was noted that after one-year of age, she gradually regressed and became 


\begin{tabular}{|l|c|c|c|c|}
\hline \multicolumn{5}{|c|}{ SEIZURE LOG } \\
\hline Day & Scalar Energy Rx & \# of seizures & $\begin{array}{l}\text { Min Duration } \\
\text { (seconds) }\end{array}$ & $\begin{array}{l}\text { Max duration } \\
\text { (seconds) }\end{array}$ \\
\hline Pre Rx Day -8 & & 10 & 5 & 20 \\
\hline Pre Rx Day -7 & & 6 & 10 & 20 \\
\hline Pre Rx Day -6 & & 8 & 10 & 20 \\
\hline Pre Rx Day -5 & & 8 & 10 & 20 \\
\hline Pre Rx Day -4 & & 9 & 10 & 20 \\
\hline Pre Rx Day -3 & & 8 & 5 & 20 \\
\hline Pre Rx Day -2 & & 6 & 10 & 20 \\
\hline Pre Rx Day -1 & & 6 & 10 & 20 \\
\hline Day 1 & SESSION 1 & 6 & 5 & 6 \\
\hline Day 2 & SESSION 2 & 5 & 4 & 5 \\
\hline Day 3 & & 5 & 4 & 5 \\
\hline Day 4 & SESSION 3 & 0 & 0 & 0 \\
\hline Day 5 & & 1 & 1 & 1 \\
\hline Day 6 & & 5 & 4 & 5 \\
\hline Day 7 & & 6 & 5 & 8 \\
\hline Day 8 & & 5 & 5 & 8 \\
\hline Day 9 & & 5 & 4 & 8 \\
\hline Day 10 & SESSION 4 & 4 & 4 & 5 \\
\hline Day 11 & SESSION 5 & 6 & 5 & 8 \\
\hline Day 12 & & 4 & 4 & 5 \\
\hline Day 13 & & 2 & 1 & 45 \\
\hline Day 14 & SESSION 6 & 1 & 1 & 1 \\
\hline Day 15 & SESSION 7 & 2 & 1 & 1 \\
\hline Day 16 & & 6 & 1 & 6 \\
\hline & Log not done in this interval & & \\
\hline Day 56 & SESSION 22 & 5 & 1 & 2 \\
\hline Day 57 & & 4 & 1 & 2 \\
\hline Day 58 & SESSION 23 & 4 & 1 & 1 \\
\hline
\end{tabular}

Figure 1. The seizure log.

withdrawn and introverted, becoming less responsive to social interaction.

At about age 23 months, her mother noticed one or two second "head bobs" or brief periods of unresponsiveness or breaks in consciousness. These periods became more and more frequent and the patient was taken to a pediatric neurologist. The neurologist diagnosed absence seizures and prescribed tegretol.

Two months later, the patient suffered a tonic-clonic seizure lasting approximately 3 minutes. Medication was adjusted but while duration of the seizures were shorter, seizure frequency increased over the next year. She also began to experience "drop" episodes when patient would collapse and be unresponsive for a few seconds. Over the next year, she was placed on topamax, keppra, lamictal, and micelene. The patient stabilized with grand mal seizures 6 to 8 times per day, each lasting 10 to 20 seconds in duration.

At the time of the beginning of the EES treatment, she had not had a seizure-free day in two years. The pattern of 6 to 8 seizures per day had persisted for a period of approximately 1 year prior to first presentation.

Her mother stated, "on a good day, she would have about 2 - 3 "grand mals" daily, and 8 - 10 "drop" seizures lasting 1 to 2 seconds before using the scalar field therapy."

\section{Physical exam}

On initial examination, patient was in a stroller and noncommunicative. Eye contact was present only upon repeated request from her mother and only for a few seconds. She was observed to be looking randomly moving about the room or focused on an object of interest. She was found to be alert but oriented to person but not time or place. She appeared to have normal movement and was able to handle toys and have reasonable eye-hand coordination. She responded to commands only after repeated requests by her mother.

\section{Therapy}

The therapy consisted of the patient being placed for one or two hour sessions in the scalar field generated by the EESystem (EES) manufactured by Energy Enhancement System, LLC. The patient was accompanied by and supervised by her mother and received two to three scalar field sessions per week. During the sessions, the patient sat in her stroller or on the floor or lay down on a massage table. She was allowed to play with toys, move about within the room, sleep and eat or drink as necessary. There was no equipment or supplies that touch the patient during the treatment. Patient's mother was 
asked to keep a log of seizures one week prior to the therapy and to continue keeping a $\log$ to see if there was any difference in number or duration of seizures.

Scalar fields were first described by James Clerk Maxwell in the mid-1800's and further explored by Nikola Tesla, the father of the alternating current system widely in use today. The definition of scalar quantities are those that are fully described by a magnitude (or numerical value) alone. Vector quantities are those that are fully described by both a magnitude and a direction. Electromagnetic fields are vector quantities which have both magnitude and direction such as pointing north or south. So, by definition, scalar energy fields are distinguished from ordinary electromagnetic fields in that have they have energy but no directional component.

The EES is described to be a "scalar field" generating system that is composed of 4 or more scalar field generators, each resembling a computer with a monitor screen. They are set up in diametrically opposed pairs around the corners of a room. Conceptually it is somewhat akin to the use of pulsed electromagnetic frequencies for bone healing. However, this system apparently cancels electromagnetic fields in the chamber by having each opposing generator send identical signals towards each other which negates each other's directional component. This purportedly allows the remaining energy to exist as a scalar field.

\section{Mechanism}

Scalar fields have been reportedly used for healing since the 1960 's by various proponents using various different scalar field generators [5]. The scalar field purportedly enhances the metabolism of the cell by being set to pulse at a frequency harmonious to living cells and restores optimal potential difference between the inside and the outside of each cell. This in turn would help the cells to function optimally, eliminate toxins more efficiently and repair itself more quickly.

Studies on its effect on healing suggest that there is a very real neurological effect on meditation and antidepressant medication [6]. A Stanford researcher published data that suggests that scalar fields can cause a reduction in noradrenaline reuptake [7]. This is similar to the action of some anti-depressant medications.

Unpublished reports have shown dark-field microscopy photographs of blood cells showing a remarkable reduction of rouleaux formation after one hour exposure to the EES suggesting that it may reduce blood viscosity. Reports from an oncologist using the EES suggests that there may be a detoxification effect that may be occurring as well.

\section{Outcome}

On the week prior to treatment, patient's seizure log indicated 6 to 10 seizures per day of 5 to 20 second duration. After the second treatment, patient's mother noted a reduction in the number and duration of seizures. After the third treatment (day 4 since the beginning of treatment) the patient had a seizure-free day for the first time in a year and a half according to her mother. She continued with two to three treatments per week, each lasting two hours long with the number and duration of the seizures gradually diminishing (Fig. 1).

On day 13, two days after her 5th treatment, she had just two seizures, a one-second partial seizure and an unusually long 45 second tonic-clonic seizure. Thereafter, the duration of the seizures diminished to 1 or 2 second partial seizures. The number of these tonic-clonic seizures declined to less than one per week with only the occurrence of 2 to 6 partial or atonic seizures of short duration. During the course of treatment and thereafter, there was no change in her medication. No adverse side-effects were observed. Six months later, she typically had no tonic-clonic seizures and just 4 to 5 partial or atonic seizures per day, each lasting no more than 1 to 2 seconds.

\section{Discussion}

The patients improvement associated with the therapy is substantial and remarkable. Going from 6 to 8 generalized tonicclonic seizures per day, to virtually none in a period of a few weeks is quite remarkable especially when one considers that there was no change in medication. Certainly, this is not a cure, as she presently has a number of 1 to 2 second partial or atonic seizures per day, and she is still on prescription medication. It is possible that the reduction in seizures may be a coincidental reduction as the patient ages and mylenization progresses. However, there is unlikely to be a spontaneous remission in this child who has already been documented to have autism and intractable seizures with medication [8]. Moreover, placebo effect is unlikely in a child who is unaware of the treatment. These considerations and the sudden and sustained drop in number of seizures suggests strongly that the scalar field treatments are causing this improvement.

The mitigation of seizure disorder through the use of the EES may be due to its apparent effect on neuronal function or through its effect on blood circulation or other cellular effects. The in vitro finding of the effect of scalar fields on noradrenaline uptake could explain a change in seizure threshold, for example. These mechanisms along with other possible mechanisms should be tested because of their potential for treatment in seizures and other related diseases.

\section{Conclusion}

This case study suggests that the scalar field therapy delivered by the EES reduced the number and severity of seizures in this child without adding medication. It remains to be seen if scalar field therapy will achieve similar outcomes in other children and whether the effect is long-standing. It also re- 
mains to be seen if the improvement is substantial enough to reduce the need for medication.

This promising case makes it important that more studies should be conducted to study innovative scalar field treatments. Studies should be done to: 1) explore in a double blind study the relationship between the therapy and the outcome, 2) define the mechanism of the anti-seizure effect, 3 ) define the long term results of treatment, 4) document whether any adverse side effects may result, and 5) explore any other conditions that may be remediated with scalar field therapy.

\section{Conflict of Interest}

Dr. Apau-Ludlum and Dr. Harrigan have no conflict of interest to report. The second author, Terry Shintani, MD, JD, MPH set up an LLC which he controls for the purpose of holding the Energy Enhancement System scalar field generator for limitation of liability purposes and to pay for the operation of the System and related expenses. He receives no pay from the LLC.

\section{Grant Funds}

None.

\section{References}

1. Canitano R. Epilepsy in autism spectrum disorders. Eur Child Adolesc Psychiatry. 2007;16(1):61-66.

2. American Academy of Pediatrics: The pediatrician's role in the diagnosis and management of autistic spectrum disorder in children. Pediatrics. 2001;107(5):12211226.

3. Wheless JW, Clarke DF, Carpenter D. Treatment of pediatric epilepsy: expert opinion, 2005. J Child Neurol. 2005;20 Suppl 1:S1-56; quiz S59-60.

4. Alvarez N, Besag F, Iivanainen M. Use of antiepileptic drugs in the treatment of epilepsy in people with intellectual disability. J Intellect Disabil Res. 1998;42 Suppl $1: 1-15$

5. Puharich A. Fourteenth United States Psychotronic Association Conference on Bioenergy, Dayton, OH, 1988.

6. Byrd E. Why Life Responds To and Radiates $10 \mathrm{~Hz}$, Journal of the United States Phychotronics Association, 1989;2(1).

7. Rein, Glen. Biological Interactions with Scalar Energy - Cellular Mechanisms of Actions, Proceedings Seventh International Association Psychotronics Research, December, 1988.

8. Kwan P, Sander JW. The natural history of epilepsy: an epidemiological view. J Neurol Neurosurg Psychiatry. 2004;75(10):1376-1381. 